\title{
Finite-Time Synchronization for Heterogeneous Complex Networks with Time-Varying Delays
}

\author{
Yicong Ma ${ }^{1}$, Yali Tai ${ }^{2}$ \\ ${ }^{1}$ School of Science, Jimei University, Xiamen, China \\ ${ }^{2}$ Chengyi University College, Jimei University, Xiamen, China \\ Email: 32141045@qq.com
}

How to cite this paper: Ma, Y.C. and Tai, Y.L. (2020) Finite-Time Synchronization for Heterogeneous Complex Networks with Time-Varying Delays. Applied Mathematics, 11, 1000-1012. https://doi.org/10.4236/am.2020.1110066

Received: September 23, 2020

Accepted: October 23, 2020

Published: October 26, 2020

Copyright (c) 2020 by author(s) and Scientific Research Publishing Inc. This work is licensed under the Creative Commons Attribution International License (CC BY 4.0).

http://creativecommons.org/licenses/by/4.0/

(c) (i) Open Access

\begin{abstract}
This paper studies the problem of finite-time synchronization for a class of heterogeneous complex networks which not only have node time-varying delays and coupled time-varying delays but also contain uncertain disturbance. An appropriate controller is designed such that this type of network can be synchronized within a finite time. By constructing a proper Lyapunov function and using the finite-time stability theory, the sufficient conditions for the network to achieve finite-time synchronization are given and the finite time is estimated. Finally, the conclusions obtained are extended to the case of homogeneous complex networks with time-varying delays and uncertain disturbance.
\end{abstract}

\section{Keywords}

Finite-Time Synchronization, Heterogeneous, Time-Varying Delays, Uncertain Disturbance

\section{Introduction}

In the past few decades, complex networks (CNs) have received increasing attention due to their applications in various fields including food webs, communication networks, the Internet and so on. Much effort has been devoted to the control and synchronization of CNs, since it can describe many phenomena in nature and human society, and has many potential applications [1] [2] [3]. So far, some fruitful results about the synchronization of CNs have been obtained [4] [5] [6] [7]. The research on synchronization and synchronization control of CNs is very meaningful. In order to achieve system synchronization, many methods such as impulse control [8] [9] [10], adaptive control [11] [12] and their combination [13] [14] are widely used in chaotic systems. Due to noise, limited 
signal transmission and other reasons, time delays are common in nature and they can also change with time. The time-varying delays in complex networks usually include node time delays and coupling time delays [15] [16] [17].

The complex dynamic network model studied in [18] has node time delay and coupling time delay, but it studies the asymptotic synchronization of complex dynamic network, which means that the complex network achieves synchronization in infinite time. In practical applications, the synchronization of complex dynamic networks usually needs to be achieved within a limited time. Therefore, in recent years, the finite-time synchronization of complex dynamic networks has been paid attention to by many scholars, and some research results have been obtained [19]-[24]. In [24] the finite-time synchronization of complex dynamic networks with time delay is studied. The nodes of the complex networks studied above all have the same dynamic system, but in the real world, there are differences between the network nodes, and the dynamic systems of the network nodes are not exactly the same, that is, the expression of the state function for different nodes is different. The synchronization control of heterogeneous complex network has also attracted the attention of scholars [25] [26] [27]. Reference [28] studied the finite-time synchronization problems of heterogeneous complex networks, but did not discuss the situation of nodes with time delay and coupling time delay.

According to the points discussed above, this paper aims to further investigate finite-time synchronization of heterogeneous complex networks with time-varying delays and uncertain disturbance. Based on the finite-time stability theory, by designing an appropriate controller and constructing proper Lyapunov function this kind of node heterogeneous time-delayed complex networks can achieve finite-time synchronization. And we can obtain the sufficient conditions for the finite-time synchronization. Then, the results obtained are extended to the case of homogeneous complex networks with time-varying delays and uncertain disturbance.

The remainder of this paper is organized as follows. Section 2 presents the model and necessary preliminaries. In Section 3 the main results are given. Section 4 is the conclusions.

\section{Model and Preliminaries}

Consider a heterogeneous complex network with time-varying delay and linearly coupled by $N$ different nodes, the state equation of the $i$ - $t$ node $(i=1,2, \cdots, N)$ is

$$
\dot{x}_{i}(t)=f_{i}\left(t, x_{i}(t), x_{i}\left(t-\tau_{1}(t)\right)\right)+p_{i}\left(t, x_{i}(t)\right)+c \sum_{j=1}^{N} a_{i j} G x_{j}\left(t-\tau_{2}(t)\right)
$$

$x_{i}(t)=\left(x_{i 1}(t), x_{i 2}(t), \cdots, x_{i n}(t)\right)^{\mathrm{T}} \in R^{n}$ is the state variable of the $i-t h$ node at time $t, \quad f\left(t, x_{i}(t), x_{i}\left(t-\tau_{1}(t)\right)\right): R^{+} \times R^{n} \times R^{n} \rightarrow R^{n}$ is the smooth vector function of the $i$-th dynamic system; $p_{i}\left(t, x_{i}(t)\right): R^{+} \times R^{n} \rightarrow R^{n}$ is the uncertain disturbance term of the $i$-th dynamic system and it is a bounded function; 
$0 \leq \tau_{1}(t) \leq \tau_{1}, 0 \leq \tau_{2}(t) \leq \tau_{2}$ are respectively node time-varying delay and coupling time delay, where $\tau_{1}, \tau_{2}$ are known constants; $c \in R^{+}$is the network coupling strength; external coupling matrix $A=\left(a_{i j}\right) \in R^{N \times N}$ represents the external coupling constant matrix between nodes, where the matrix $A$ does not need to be a symmetric matrix, if there is a connection between node $i$ and node $j$, then $a_{i j}>0$, otherwise $a_{i j}=0(i \neq j)$, and the diagonal elements $a_{i i}=-\sum_{j=1, j \neq i}^{N} a_{i j}$, $i=1,2, \cdots, N ; G=\operatorname{diag}\left(\delta_{1}, \delta_{2}, \cdots, \delta_{n}\right) \in R^{n \times n}$ is an internal coupling matrix and $\delta_{i} \geq 0,(i=1,2, \cdots, n)$, which represents the internal topology of the network.

Definition 1 . The synchronous solution of the heterogeneous complex network (1) can be described by

$$
\dot{x}_{0}(t)=f_{0}\left(t, x_{0}(t), x_{0}\left(t-\tau_{1}(t)\right)\right)+p_{0}\left(t, x_{0}(t)\right)
$$

It can be a balance point, periodic trajectory or chaotic trajectory.

Definition 2. The node error of heterogeneous complex network (1) is

$$
e_{i}(t)=x_{i}(t)-x_{0}(t), \quad i=1,2, \cdots, N
$$

The finite-time synchronization problem of the heterogeneous complex network (1) can be defined as follows:

If there is a time $t^{*}>0$ such that

$$
\lim _{t \rightarrow t^{*}}\left\|e_{i}(t)\right\|=0 \text { and }\left\|e_{i}(t)\right\| \equiv 0 \text { for } t>t^{*}, i=1,2, \cdots, N,
$$

where $t^{*}$ is called the settling time, then the complex network is synchronized with the synchronization solution $x_{0}(t)$ in a finite time.

In order to complete the finite-time synchronization of the heterogeneous complex network (1), the controller $u_{i}(t) \in R^{n}$ needs to be applied to the nodes of the complex network (1). The design is as follows:

$$
\begin{aligned}
u_{i}(t)= & -d_{i} e_{i}(t)-\eta(t) \operatorname{SIGN}\left(e_{i}(t)\right) \\
& -k\left[\operatorname{sign}\left(e_{i}(t)\right)\left|e_{i}(t)\right|^{\beta}+\left(k_{1} \int_{t-\tau_{1}(t)}^{t} e_{i}^{\mathrm{T}}(s) e_{i}(s) \mathrm{d} s\right)^{\frac{1+\beta}{2}} \frac{e_{i}(t)}{\left\|e_{i}(t)\right\|^{2}}\right. \\
& \left.+\left(k_{2} \int_{t-\tau_{2}(t)}^{t} e_{i}^{\mathrm{T}}(s) e_{i}(s) \mathrm{d} s\right)^{\frac{1+\beta}{2}} \frac{e_{i}(t)}{\left\|e_{i}(t)\right\|^{2}}\right]
\end{aligned}
$$

where $\operatorname{sign}(\cdot)$ represents a symbolic function, $d_{i}>0, k_{1}>0, k_{2}>0$ are undetermined constants, $k$ is a given positive constant, $\beta \in R$ and satisfies $0 \leq \beta<1$.

In Equation (4),

$$
\begin{gathered}
\operatorname{SIGN}\left(e_{i}(t)\right)=\left[\operatorname{sign}\left(e_{i 1}(t)\right), \operatorname{sign}\left(e_{i 2}(t)\right), \cdots, \operatorname{sign}\left(e_{i n}(t)\right)\right]^{\mathrm{T}} \\
\operatorname{sign}\left(e_{i}(t)\right)=\operatorname{diag}\left[\operatorname{sign}\left(e_{i 1}(t)\right), \operatorname{sign}\left(e_{i 2}(t)\right), \cdots, \operatorname{sign}\left(e_{i n}(t)\right)\right] \\
\left|e_{i}(t)\right|^{\beta}=\left[\left|e_{i 1}(t)\right|^{\beta},\left|e_{i 2}(t)\right|^{\beta}, \cdots,\left|e_{i n}(t)\right|^{\beta}\right]^{\mathrm{T}}
\end{gathered}
$$

So the complex network (1) with controller $u_{i}(t)$ can be expressed as: 


$$
\begin{aligned}
\dot{x}_{i}(t)= & f_{i}\left(t, x_{i}(t), x_{i}\left(t-\tau_{1}(t)\right)\right)+p_{i}\left(t, x_{i}(t)\right) \\
& +c \sum_{j=1}^{N} a_{i j} G x_{j}\left(t-\tau_{2}(t)\right)+u_{i}(t)
\end{aligned}
$$

From Equation (2), Equation (3) and Equation (8) the network error dynamic equation of the system can be derived as

$$
\begin{aligned}
\dot{e}_{i}(t)= & f_{i}\left(t, x_{i}(t), x_{i}\left(t-\tau_{1}(t)\right)\right)-f_{0}\left(t, x_{0}(t), x_{0}\left(t-\tau_{1}(t)\right)\right) \\
& +p_{i}\left(t, x_{i}(t)\right)-p_{0}\left(t, x_{0}(t)\right)+c \sum_{j=1}^{N} a_{i j} G e_{j}\left(t-\tau_{2}(t)\right)+u_{i}(t) \\
= & F_{i}\left(t, e_{i}(t), e_{i}\left(t-\tau_{1}(t)\right)\right)+P_{i}\left(t, e_{i}(t)\right) \\
& +c \sum_{j=1}^{N} a_{i j} G e_{j}\left(t-\tau_{2}(t)\right)+u_{i}(t)
\end{aligned}
$$

The assumptions and lemmas needed to prove the finite-time synchronization of the heterogeneous complex network (1) are given below.

Assumption 1. [29] For any $x(t), y(t) \in R^{n}$, there are positive constants $L_{1}, L_{2}$, satisfying the inequality

$$
\begin{aligned}
& (x(t)-y(t))^{\mathrm{T}}[f(t, x(t), x(t-\tau(t)))-f(t, y(t), y(t-\tau(t)))] \\
& \leq L_{1}[x(t)-y(t)]^{\mathrm{T}}[x(t)-y(t)] \\
& +L_{2}[x(t-\tau(t))-y(t-\tau(t))]^{\mathrm{T}}[x(t-\tau(t))-y(t-\tau(t))]
\end{aligned}
$$

Assumption 2. $0 \leq \dot{\tau}_{1}(t) \leq h_{1}<1, \quad 0 \leq \dot{\tau}_{2}(t) \leq h_{2}<1$ where $h_{i}(i=1,2)$ are known constants.

Lemma 1. [30] For any two vectors $x, y \in R^{n}$, the following inequality holds

$$
2 x^{\mathrm{T}} y \leq x^{\mathrm{T}} x+y^{\mathrm{T}} y .
$$

Assumption 3. [31] There is a time-varying bounded function $\mu(t)>0$, such that the following inequality holds

$$
\left\|f_{i}(t, x(t))-f_{0}(t, x(t))\right\|<\mu(t), \quad i=1,2, \cdots, N .
$$

Assumption 4. There exists a positive constant $p_{\max }$ for the uncertain disturbance term $p_{i}\left(t, x_{i}(t)\right), i=0,1,2, \cdots, N$, such that $\left\|p_{i}\left(t, x_{i}(t)\right)\right\| \leq p_{\max }$.

Lemma 2. [32] For $\forall a_{i} \in R, i=1,2, \cdots, n$, if $p, q \in R, 0<p \leq 1,0<q<2$, then we have

$$
\begin{gathered}
\left|a_{1}\right|^{q}+\left|a_{2}\right|^{q}+\cdots+\left|a_{n}\right|^{q} \geq\left(\left|a_{1}\right|^{2}+\left|a_{2}\right|^{2}+\cdots+\left.\left|a_{n}\right|^{2}\right|^{\frac{q}{2}}\right. \\
\left(\left|a_{1}\right|+\left|a_{2}\right|+\cdots+\left|a_{n}\right|\right)^{p} \leq\left|a_{1}\right|^{p}+\left|a_{2}\right|^{p}+\cdots+\left|a_{n}\right|^{p} .
\end{gathered}
$$

Lemma 3. [33] Assume that a continuous, positive-definite function $V(t)$ satisfies the following differential inequality:

$$
\frac{\mathrm{d} V(t)}{\mathrm{d} t} \leq-\varepsilon V^{\alpha}(t), \quad \forall t \geq 0, V(0) \geq 0,
$$

where $\alpha, \varepsilon$ are positive constants and $0<\alpha<1$, then 


$$
\left\{\begin{array}{l}
V^{1-\alpha}(t) \leq V^{1-\alpha}(0)-\varepsilon(1-\alpha) t, \quad 0<t<t^{*} \\
V(t) \equiv 0, \quad t>t^{*}=\frac{V^{1-\alpha}(0)}{\varepsilon(1-\alpha)}
\end{array}\right.
$$

\section{Main Results}

In this section, by using Lyapunov function and finite-time stability theory, the authors focus on investigating finite-time synchronization of the heterogeneous complex networks with time-varying delay and uncertain disturbance. The main results are given by the following theorem:

Theorem 1 . If there are positive constants $d_{i}(i=1,2, \cdots, N), k_{1}, k_{2}$ satisfy the following conditions

$$
\begin{gathered}
\left(L_{1}+\frac{k_{1}}{2}+\frac{k_{2}}{2}\right) I-\left(D \otimes I_{n}\right)+\frac{c}{2} B B^{\mathrm{T}} \leq 0 \\
L_{2}-\frac{k_{1}}{2}\left(1-h_{1}\right) \leq 0 \\
\frac{c}{2}-\frac{k_{2}}{2}\left(1-h_{2}\right) \leq 0 \\
2 p_{\max }+\mu(t)-\eta(t) \leq 0
\end{gathered}
$$

where $D=\operatorname{diag}\left(d_{1}, d_{2}, \cdots, d_{N}\right)>0, \quad A^{S}=\frac{A+A^{\mathrm{T}}}{2}, \quad B=A^{S} \otimes G$, then under the premise of satisfying Assumptions 1 - 4, the controlled network (8) of the nodes applying the controller (4) can be synchronized in finite time. The settling time is estimated as $t^{*}=\frac{V^{\frac{1-\beta}{2}}(0)}{k 2^{\frac{\beta-1}{2}}(1-\beta)}$.

Proof: Construct Lyapunov function,

$$
\begin{aligned}
V(t) & =\frac{1}{2} \sum_{i=1}^{N} e_{i}^{\mathrm{T}}(t) e_{i}(t)+\frac{k_{1}}{2} \sum_{i=1}^{N} \int_{t-\tau_{1}(t)}^{t} e_{i}^{\mathrm{T}}(s) e_{i}(s) \mathrm{d} s+\frac{k_{2}}{2} \sum_{i=1}^{N} \int_{t-\tau_{2}(t)}^{t} e_{i}^{\mathrm{T}}(s) e_{i}(s) \mathrm{d} s \\
& =V_{1}(t)+V_{2}(t)+V_{3}(t)
\end{aligned}
$$

Take the derivative of $V(t)$ along the error system (9),

$$
\dot{V}(t)=\dot{V}_{1}(t)+\dot{V}_{2}(t)+\dot{V}_{3}(t)
$$

The derivative of $V_{1}(t)$ can be calculated as

$$
\begin{aligned}
\dot{V}_{1}(t)= & \sum_{i=1}^{N} e_{i}^{\mathrm{T}}(t)\left[F_{i}\left(t, e_{i}(t), e_{i}\left(t-\tau_{1}(t)\right)\right)+P_{i}\left(t, e_{i}(t)\right)\right. \\
& \left.+c \sum_{j=1}^{N} a_{i j} G e_{j}\left(t-\tau_{2}(t)\right)+u_{i}(t)\right] \\
= & \sum_{i=1}^{N} e_{i}^{\mathrm{T}}(t) F_{i}\left(t, e_{i}(t), e_{i}\left(t-\tau_{1}(t)\right)\right)+\sum_{i=1}^{N} e_{i}^{\mathrm{T}}(t) P_{i}\left(t, e_{i}(t)\right) \\
& +c \sum_{i=1}^{N} e_{i}^{\mathrm{T}}(t) \sum_{j=1}^{N} a_{i j} G e_{j}\left(t-\tau_{2}(t)\right)+\sum_{i=1}^{N} e_{i}^{\mathrm{T}}(t) u_{i}(t) \\
= & W_{1}+W_{2}+W_{3}+W_{4}
\end{aligned}
$$


In Equation (16),

$$
\begin{aligned}
& W_{1}=\sum_{i=1}^{N} e_{i}^{\mathrm{T}}(t) F_{i}\left(t, e_{i}(t), e_{i}\left(t-\tau_{1}(t)\right)\right) \\
& W_{2}=\sum_{i=1}^{N} e_{i}^{\mathrm{T}}(t) P_{i}\left(t, e_{i}(t)\right) \\
& W_{3}=c \sum_{i=1}^{N} e_{i}^{\mathrm{T}}(t) \sum_{j=1}^{N} a_{i j} G e_{j}\left(t-\tau_{2}(t)\right) \\
& W_{4}=\sum_{i=1}^{N} e_{i}^{\mathrm{T}}(t) u_{i}(t)
\end{aligned}
$$

$W_{1}$ can be rewritten as

$$
\begin{aligned}
W_{1}= & \sum_{i=1}^{N} e_{i}^{\mathrm{T}}(t)\left[f_{i}\left(t, x_{i}(t), x_{i}\left(t-\tau_{1}(t)\right)\right)-f_{0}\left(t, x_{0}(t), x_{0}\left(t-\tau_{1}(t)\right)\right)\right] \\
= & \sum_{i=1}^{N} e_{i}^{\mathrm{T}}(t)\left[f_{i}\left(t, x_{i}(t), x_{i}\left(t-\tau_{1}(t)\right)\right)-f_{i}\left(t, x_{0}(t), x_{0}\left(t-\tau_{1}(t)\right)\right)\right. \\
& \left.+f_{i}\left(t, \quad x_{0}(t), x_{0}\left(t-\tau_{1}(t)\right)\right)-f_{0}\left(t, x_{0}(t), x_{0}\left(t-\tau_{1}(t)\right)\right)\right] \\
= & W_{11}+W_{12}
\end{aligned}
$$

where

$$
\begin{aligned}
& W_{11}=\sum_{i=1}^{N} e_{i}^{\mathrm{T}}(t)\left[f_{i}\left(t, x_{i}(t), x_{i}\left(t-\tau_{1}(t)\right)\right)-f_{i}\left(t, x_{0}(t), x_{0}\left(t-\tau_{1}(t)\right)\right)\right] \\
& W_{12}=\sum_{i=1}^{N} e_{i}^{\mathrm{T}}(t)\left[f_{i}\left(t, x_{0}(t), x_{0}\left(t-\tau_{1}(t)\right)\right)-f_{0}\left(t, x_{0}(t), x_{0}\left(t-\tau_{1}(t)\right)\right)\right]
\end{aligned}
$$

Let $e(t)=\left[e_{1}^{\mathrm{T}}(t), e_{2}^{\mathrm{T}}(t), \cdots, e_{N}^{\mathrm{T}}(t)\right]^{\mathrm{T}}$ and using Assumption 1, we have

$$
\begin{aligned}
W_{11} & \leq \sum_{i=1}^{N}\left[L_{1} e_{i}^{\mathrm{T}}(t) e_{i}(t)+L_{2} e_{i}^{\mathrm{T}}\left(t-\tau_{1}(t)\right) e_{i}\left(t-\tau_{1}(t)\right)\right] \\
& =L_{1} e^{\mathrm{T}}(t) e(t)+L_{2} e^{\mathrm{T}}\left(t-\tau_{1}(t)\right) e\left(t-\tau_{1}(t)\right)
\end{aligned}
$$

Using Assumption 3 and the formula $\left\|e_{i}(t)\right\|_{2}=\left(\sum_{j=1}^{n}\left|e_{i j}(t)\right|^{2}\right)^{\frac{1}{2}}$, we obtain

$$
\begin{aligned}
W_{12} & \leq \sum_{i=1}^{N}\left\|e_{i}(t)\right\|_{2}\left\|f_{i}\left(t, x_{0}(t), x_{0}\left(t-\tau_{1}(t)\right)\right)-f_{0}\left(t, x_{0}(t), x_{0}\left(t-\tau_{1}(t)\right)\right)\right\|_{2} \\
& \leq \mu(t) \sum_{i=1}^{N}\left\|e_{i}(t)\right\|_{2}
\end{aligned}
$$

By using Assumption 4, we can get

$$
\begin{aligned}
W_{2} & =\sum_{i=1}^{N} e_{i}^{\mathrm{T}}(t)\left[p_{i}\left(t, x_{i}(t)\right)-p_{0}\left(t, x_{0}(t)\right)\right] \\
& \leq \sum_{i=1}^{N}\left\|e_{i}(t)\right\|_{2}\left\|p_{i}\left(t, x_{i}(t)\right)-p_{0}\left(t, x_{0}(t)\right)\right\|_{2} \\
& \leq \sum_{i=1}^{N}\left\|e_{i}(t)\right\|_{2}\left(\left\|p_{i}\left(t, x_{i}(t)\right)\right\|_{2}+\left\|p_{0}\left(t, x_{0}(t)\right)\right\|_{2}\right) \\
& \leq 2 p_{\max } \sum_{i=1}^{N}\left\|e_{i}(t)\right\|_{2}
\end{aligned}
$$


Let $A^{S}=\frac{A+A^{\mathrm{T}}}{2}$, and note that $G$ is a diagonal matrix, by utilizing the quality of the Kronecker product of matrices,

$$
\begin{aligned}
W_{3} & =c(t) \sum_{i=1}^{N} e_{i}^{\mathrm{T}}(t) \sum_{j=1}^{N} a_{i j} G e_{j}\left(t-\tau_{2}(t)\right) \\
& \leq c e^{\mathrm{T}}(t)(A \otimes G) e\left(t-\tau_{2}(t)\right) \\
& =c e^{\mathrm{T}}(t)\left(\frac{A \otimes G+(A \otimes G)^{\mathrm{T}}}{2}\right) e\left(t-\tau_{2}(t)\right) \\
& =c e^{\mathrm{T}}(t)\left(A^{S} \otimes G\right) e\left(t-\tau_{2}(t)\right)
\end{aligned}
$$

Let $B$ be defined by $B=A^{S} \otimes G$, it can be obtained that

$$
W_{3} \leq c e^{\mathrm{T}}(t) B e\left(t-\tau_{2}(t)\right)
$$

From Equation (4), namely the definition of $u_{i}(t), W_{4}$ can be represented as follows:

$$
\begin{aligned}
W_{4}= & \sum_{i=1}^{N} e_{i}^{\mathrm{T}}(t)\left\{-d_{i} e_{i}(t)-\eta(t) \operatorname{SIGN}\left(e_{i}(t)\right)-k\left[\operatorname{sign}\left(e_{i}(t)\right)\left|e_{i}(t)\right|^{\beta}\right.\right. \\
& +\left(k_{1} \int_{t-\tau_{1}(t)}^{t} e_{i}^{\mathrm{T}}(s) e_{i}(s) \mathrm{d} s\right)^{\frac{1+\beta}{2}} \frac{e_{i}(t)}{\left\|e_{i}(t)\right\|^{2}} \\
& \left.\left.+\left(k_{2} \int_{t-\tau_{2}(t)}^{t} e_{i}^{\mathrm{T}}(s) e_{i}(s) \mathrm{d} s\right)^{\frac{1+\beta}{2}} \frac{e_{i}(t)}{\left\|e_{i}(t)\right\|^{2}}\right]\right\} \\
= & W_{41}+W_{42}+W_{43}
\end{aligned}
$$

Define $D=\operatorname{diag}\left(d_{1}, d_{2}, \cdots, d_{N}\right)>0$, and note that

$$
\sum_{i=1}^{N} e_{i}^{\mathrm{T}}(t) \operatorname{SIGN}\left(e_{i}(t)\right)=\sum_{i=1}^{N} \sum_{j=1}^{n}\left|e_{i j}(t)\right|,
$$

we have from Equation (22),

$$
\begin{aligned}
W_{41} & =\sum_{i=1}^{N} e_{i}^{\mathrm{T}}(t)\left[-d_{i} e_{i}(t)-\eta(t) \operatorname{SIGN}\left(e_{i}(t)\right)\right] \\
& =-e^{\mathrm{T}}(t)\left(D \otimes I_{n}\right) e(t)-\eta(t) \sum_{i=1}^{N} e_{i}^{\mathrm{T}}(t) \operatorname{SIGN}\left(e_{i}(t)\right) \\
& \leq-e^{\mathrm{T}}(t)\left(D \otimes I_{n}\right) e(t)-\eta(t) \sum_{i=1}^{N}\left\|e_{i}(t)\right\|_{2}
\end{aligned}
$$

By using Lemma 2, we have $\left(\sum_{i=1}^{N} \sum_{j=1}^{n}\left|e_{i j}(t)\right|^{\beta+1}\right)^{\frac{1}{\beta+1}} \geq\left(\sum_{i=1}^{N} \sum_{j=1}^{n}\left|e_{i j}(t)\right|^{2}\right)^{\frac{1}{2}}$, yields

$$
\sum_{i=1}^{N} \sum_{j=1}^{n}\left|e_{i j}(t)\right|^{\beta+1} \geq\left(\sum_{i=1}^{N} \sum_{j=1}^{n}\left|e_{i j}(t)\right|^{2}\right)^{\frac{\beta+1}{2}}
$$

Combining (24) and setting $\left|e_{i}(t)\right|=\left[\left|e_{i 1}(t)\right|,\left|e_{i 2}(t)\right|, \cdots,\left|e_{i n}(t)\right|\right]^{\mathrm{T}}$, it can be obtained that 


$$
\begin{aligned}
W_{42}= & -k \sum_{i=1}^{N} e_{i}^{\mathrm{T}}(t) \operatorname{sign}\left(e_{i}(t)\right)\left|e_{i}(t)\right|^{\beta} \\
= & -k \sum_{i=1}^{N}\left|e_{i}(t)\right|^{T}\left|e_{i}(t)\right|^{\beta} \\
= & -k \sum_{i=1}^{N} \sum_{j=1}^{n}\left|e_{i j}(t)\right|^{\beta+1} \\
\leq & -k\left(\sum_{i=1}^{N} \sum_{j=1}^{n}\left|e_{i j}(t)\right|^{2}\right)^{\frac{\beta+1}{2}} \\
= & -k\left(\sum_{i=1}^{N} e_{i}^{\mathrm{T}}(t) e_{i}(t)\right)^{\frac{\beta+1}{2}} \\
W_{43}= & -k \sum_{i=1}^{N} e_{i}^{\mathrm{T}}(t)\left[\left(k_{1} \int_{t-\tau_{1}(t)}^{t} e_{i}^{\mathrm{T}}(s) e_{i}(s) \mathrm{d} s\right)^{\frac{1+\beta}{2}} \frac{e_{i}(t)}{\left\|e_{i}(t)\right\|^{2}}\right. \\
& \left.+\left(k_{2} \int_{t-\tau_{2}(t)}^{t} e_{i}^{\mathrm{T}}(s) e_{i}(s) \mathrm{d} s\right)^{\frac{1+\beta}{2}} \frac{e_{i}(t)}{\left\|e_{i}(t)\right\|^{2}}\right] \\
= & -k \sum_{i=1}^{N}\left(k_{1} \int_{t-\tau_{1}(t)}^{t} e_{i}^{\mathrm{T}}(s) e_{i}(s) \mathrm{d} s\right)^{\frac{1+\beta}{2}} \\
& -k \sum_{i=1}^{N}\left(k_{2} \int_{t-\tau_{2}(t)}^{t} e_{i}^{\mathrm{T}}(s) e_{i}(s) \mathrm{d} s\right)^{\frac{1+\beta}{2}}
\end{aligned}
$$

From (14), (25), (26) and Lemma 2, one has

$$
\begin{aligned}
W_{42}+W_{43} \leq & -k\left[\sum_{i=1}^{N} e_{i}^{\mathrm{T}}(t) e_{i}(t)+k_{1} \sum_{i=1}^{N} \int_{t-\tau_{1}(t)}^{t} e_{i}^{\mathrm{T}}(s) e_{i}(s) \mathrm{d} s\right. \\
& \left.+k_{2} \sum_{i=1}^{N} \int_{t-\tau_{2}(t)}^{t} e_{i}^{\mathrm{T}}(s) e_{i}(s) \mathrm{d} s\right]^{\frac{1+\beta}{2}} \\
= & -k(2 V)^{\frac{1+\beta}{2}}=-k 2^{\frac{1+\beta}{2}} V^{\frac{1+\beta}{2}}
\end{aligned}
$$

Thus, substituting (17), (18), (19), (21), (23), (27) into (16), the following inequality holds:

$$
\begin{aligned}
\dot{V}_{1}(t) \leq & L_{1} e^{\mathrm{T}}(t) e(t)+L_{2} e^{\mathrm{T}}\left(t-\tau_{1}(t)\right) e\left(t-\tau_{1}(t)\right)+\mu(t) \sum_{i=1}^{N}\left\|e_{i}(t)\right\|_{2} \\
& +2 p_{\max } \sum_{i=1}^{N}\left\|e_{i}(t)\right\|_{2}+c e^{\mathrm{T}}(t) B e\left(t-\tau_{2}(t)\right) \\
& -e^{\mathrm{T}}(t)\left(D \otimes I_{n}\right) e(t)-\eta(t) \sum_{i=1}^{N}\left\|e_{i}(t)\right\|_{2}-k 2^{\frac{1+\beta}{2}} V^{\frac{1+\beta}{2}}
\end{aligned}
$$

From (14) and $e(t)=\left[e_{1}^{\mathrm{T}}(t), e_{2}^{\mathrm{T}}(t), \cdots, e_{N}^{\mathrm{T}}(t)\right]^{\mathrm{T}}, \dot{V}_{2}, \dot{V}_{3}$ can be derived as follows:

$$
\begin{aligned}
\dot{V}_{2} & =\frac{k_{1}}{2} \sum_{i=1}^{N} e_{i}^{\mathrm{T}}(t) e_{i}(t)-\frac{k_{1}}{2}\left(1-\dot{\tau}_{1}(t)\right) \sum_{i=1}^{N} e_{i}^{\mathrm{T}}\left(t-\tau_{1}(t)\right) e_{i}\left(t-\tau_{1}(t)\right) \\
& =\frac{k_{1}}{2} e^{\mathrm{T}}(t) e(t)-\frac{k_{1}}{2}\left(1-\dot{\tau}_{1}(t)\right) e^{\mathrm{T}}\left(t-\tau_{1}(t)\right) e\left(t-\tau_{1}(t)\right)
\end{aligned}
$$




$$
\begin{aligned}
\dot{V}_{3} & =\frac{k_{2}}{2} \sum_{i=1}^{N} e_{i}^{\mathrm{T}}(t) e_{i}(t)-\frac{k_{2}}{2}\left(1-\dot{\tau}_{2}(t)\right) \sum_{i=1}^{N} e_{i}^{\mathrm{T}}\left(t-\tau_{2}(t)\right) e_{i}\left(t-\tau_{2}(t)\right) \\
& =\frac{k_{2}}{2} e^{\mathrm{T}}(t) e(t)-\frac{k_{2}}{2}\left(1-\dot{\tau}_{2}(t)\right) e^{\mathrm{T}}\left(t-\tau_{2}(t)\right) e\left(t-\tau_{2}(t)\right)
\end{aligned}
$$

By using Assumption 2, (28), (29), (30) and (15), we have

$$
\begin{aligned}
\dot{V}(t) \leq & e^{\mathrm{T}}(t)\left[\left(L_{1}+\frac{k_{1}}{2}+\frac{k_{2}}{2}\right) I-\left(D \otimes I_{n}\right)\right] e(t) \\
& +\left(L_{2}-\frac{k_{1}}{2}\left(1-h_{1}\right)\right) e^{\mathrm{T}}\left(t-\tau_{1}(t)\right) e\left(t-\tau_{1}(t)\right) \\
& +\left(2 p_{\max }+\mu(t)-\eta(t)\right) \sum_{i=1}^{N}\left\|e_{i}(t)\right\|_{2}+c e^{\mathrm{T}}(t) B e\left(t-\tau_{2}(t)\right) \\
& -k 2^{\frac{1+\beta}{2}} V^{\frac{1+\beta}{2}}-\frac{k_{2}}{2}\left(1-h_{2}\right) e^{\mathrm{T}}\left(t-\tau_{2}(t)\right) e\left(t-\tau_{2}(t)\right)
\end{aligned}
$$

Then by utilizing Lemma 1, it follows from (31)

$$
c e^{\mathrm{T}}(t) B e\left(t-\tau_{2}(t)\right) \leq \frac{c}{2} e^{\mathrm{T}}(t) B B^{\mathrm{T}} e(t)+\frac{c}{2} e^{\mathrm{T}}\left(t-\tau_{2}(t)\right) e\left(t-\tau_{2}(t)\right)
$$

Substituting (32) into (31) and utilizing the conditions (10), (11), (12), (13) in Theorem 1 yields

$$
\begin{aligned}
\dot{V}(t) \leq & e^{\mathrm{T}}(t)\left[\left(L_{1}+\frac{k_{1}}{2}+\frac{k_{2}}{2}\right) I-\left(D \otimes I_{n}\right)+\frac{c}{2} B B^{\mathrm{T}}\right] e(t) \\
& +\left[L_{2}-\frac{k_{1}}{2}\left(1-h_{1}\right)\right] e^{\mathrm{T}}\left(t-\tau_{1}(t)\right) e\left(t-\tau_{1}(t)\right) \\
& +\left(2 p_{\max }+\mu(t)-\eta(t)\right) \sum_{i=1}^{N}\left\|e_{i}(t)\right\|_{2} \\
& +\left[\frac{c}{2}-\frac{k_{2}}{2}\left(1-h_{2}\right)\right] e^{\mathrm{T}}\left(t-\tau_{2}(t)\right) e\left(t-\tau_{2}(t)\right)-k 2^{\frac{1+\beta}{2}} V^{\frac{1+\beta}{2}} \\
\leq & -k 2^{\frac{1+\beta}{2}} V^{\frac{1+\beta}{2}} .
\end{aligned}
$$

It is derived from Lemma 3 that, $V(t) \equiv 0$ for $t>t^{*}=\frac{V^{\frac{1-\beta}{2}}(0)}{k 2^{\frac{\beta-1}{2}}(1-\beta)}$.

Therefore, $\lim _{t \rightarrow t^{*}}\left\|e_{i}(t)\right\|=0$ and $\left\|e_{i}(t)\right\| \equiv 0$ for $t>t^{*}, i=1,2, \cdots, N$.

This completes the proof.

Finally, the conclusions obtained are extended to the case of homogeneous complex networks with the same node dynamic system.

When the nodes of the complex network have the same dynamic system where $f_{1}=f_{2}=\cdots=f_{N}=f$, the complex network (8) will be homogeneous complex network as follows:

$$
\begin{aligned}
\dot{x}_{i}(t)= & f\left(t, x_{i}(t), x_{i}\left(t-\tau_{1}(t)\right)\right)+p_{i}\left(t, x_{i}(t)\right) \\
& +c \sum_{j=1}^{N} a_{i j} G x_{j}\left(t-\tau_{2}(t)\right)+u_{i}(t)
\end{aligned}
$$


The synchronous solution can be described by

$$
\dot{x}_{0}(t)=f_{0}\left(t, x_{0}(t), x_{0}\left(t-\tau_{1}(t)\right)\right)+p_{0}\left(t, x_{0}(t)\right)
$$

Controllers in Theorem 1 are added to each node, we have error system

$$
\begin{aligned}
\dot{e}_{i}(t)= & f\left(t, x_{i}(t), x_{i}\left(t-\tau_{1}(t)\right)\right)-f_{0}\left(t, x_{0}(t), x_{0}\left(t-\tau_{1}(t)\right)\right) \\
& +p_{i}\left(t, x_{i}(t)\right)-p_{0}\left(t, x_{0}(t)\right)+c \sum_{j=1}^{N} a_{i j} G e_{j}\left(t-\tau_{2}(t)\right)+u_{i}(t) \\
= & F\left(t, e_{i}(t), e_{i}\left(t-\tau_{1}(t)\right)\right)+P_{i}\left(t, e_{i}(t)\right)+c \sum_{j=1}^{N} a_{i j} G e_{j}\left(t-\tau_{2}(t)\right)+u_{i}(t)
\end{aligned}
$$

Based on Theorem 1, we can get the sufficient conditions of network (33) to achieve synchronization in finite time.

Corollary 1: If there are positive constants $d_{i}(i=1,2, \cdots, N), k_{1}, k_{2}$ satisfy the following conditions

$$
\begin{gathered}
\left(L_{1}+\frac{k_{1}}{2}+\frac{k_{2}}{2}\right) I-\left(D \otimes I_{n}\right)+\frac{c}{2} B B^{\mathrm{T}} \leq 0 \\
L_{2}-\frac{k_{1}}{2}\left(1-h_{1}\right) \leq 0 \\
\frac{c}{2}-\frac{k_{2}}{2}\left(1-h_{2}\right) \leq 0 \\
2 p_{\max }-\eta(t) \leq 0
\end{gathered}
$$

where $D=\operatorname{diag}\left(d_{1}, d_{2}, \cdots, d_{N}\right)>0, \quad A^{S}=\frac{A+A^{\mathrm{T}}}{2}, \quad B=A^{S} \otimes G$, then under the premise of satisfying assumptions $1-4$, the controlled network (33) of the node applying the controller (4) can be synchronized in finite time. The settling time is estimated as $t^{*}=\frac{V^{\frac{1-\beta}{2}}(0)}{k 2^{\frac{\beta-1}{2}}(1-\beta)}$.

The proof of Corollary 1 is similar to the proof of Theorem 1 .

\section{Conclusion}

In this paper, the finite-time synchronization problem of a class of heterogeneous complex networks with node time-varying delays and coupled time-varying delays and uncertain disturbance is considered. By designing an appropriate controller and using Lyapunov function and the finite-time stability theory, the sufficient conditions formulated by a set of inequalities are derived to guarantee that all the node systems achieve synchronization with the synchronous solution in a finite settling time. Specially, the above conclusions can be applied to the case of homogeneous complex networks with time-varying delays and uncertain disturbance.

\section{Acknowledgements}

The authors would like to thank the editors and the reviewers for their valuable comments and suggestions. This work was supported by the Fujian Provincial 
Young and Middle-aged Teacher Education Research Project (JT180871).

\section{Conflicts of Interest}

The authors declare no conflicts of interest regarding the publication of this paper.

\section{References}

[1] Ott, E. (2002) Chaos in Dynamical Systems. 2nd Edition, Cambridge University Press, Cambridge. https://doi.org/10.1017/CBO9780511803260

[2] Khan, A. and Shahzad, M. (2013) Synchronization of Circular Restricted Three Body Problem with Lorenz Hyper Chaotic System Using a Robust Adaptive Sliding Mode Controller. Complexity, 18, 58-64. https://doi.org/10.1002/cplx.21459

[3] Zhou, J., Lv, J.A. and Lu, J.H. (2008) Pining Adaptive Synchronization of a General Complex Dynamical Network. Automatica, 44, 996-1003. https://doi.org/10.1016/j.automatica.2007.08.016

[4] Lv, J.H., Leung, H. and Chen, G. (2004) Complex Dynamical Networks: Modelling, Synchronization and Control. Dynamics of Continuous Discrete and Impulsive, 11, 70-77.

[5] Li, X., Wang, X.F. and Chen, G.R. (2004) Pinning a Complex Dynamical Network to Its Equilibrium. IEEE Transactions on Circuits Systems I: Regular Papers, 51, 2074-2087. https://doi.org/10.1109/TCSI.2004.835655

[6] Yu, W.W., Chen, G.R. and Lv, J.H. (2009) On Pinning Synchronization of Complex Dynamical Networks. Automatica, 45, 429-435.

https://doi.org/10.1016/j.automatica.2008.07.016

[7] Lv, J.H., Yu, X.H. and Chen, G.R. (2004) Chaos Synchronization of General Complex Dynamical Networks. Physica A: Statistical Mechanics and Its Applications, 334, 281-302. https://doi.org/10.1016/j.physa.2003.10.052

[8] Liu, B., Liu, X.Z., Chen, G.R. and Wang, H.Y. (2005) Robust Impulsive Synchronization of Un-certain Dynamical Networks. IEEE Transactions on Circuits Systems. I: Regular Papers, 52, 1431-1441. https://doi.org/10.1109/TCSI.2005.851708

[9] Jiang, H.B. and Bi, Q.S. (2010) Impulsive Synchronization of Networked Nonlinear Dynamical Systems. Physical Review Letters, 374, 2723-2729. https://doi.org/10.1016/j.physleta.2010.04.063

[10] Yang, X.S. and Yang, Z.C. (2014) Synchronization of TS Fuzzy Complex Dynamical Networks with Time-Varying Impulsive Delays and Stochastic Effects. Fuzzy Sets and Systems, 235, 25-43. https://doi.org/10.1016/j.fss.2013.06.008

[11] Yang, L.X. and Jiang, J. (2014) Adaptive Synchronization of Drive-Response Fractional-Order Complex Dynamical Networks with Uncertain Parameters. Communications in Nonlinear Science and Numerical Simulation, 19, 1496-1506. https://doi.org/10.1016/j.cnsns.2013.09.021

[12] Liu, H., Chen, J., Lu, J.A. and Cao, M. (2010) Generalized Synchronization in Complex Dynamical Networks via Adaptive Couplings. Physica A: Statistical Mechanics and Its Applications, 389, 1759-1770. https://doi.org/10.1016/j.physa.2009.12.035

[13] Li, K. and Lai, C.H. (2008) Adaptive-impulsive Synchronization of Uncertain Complex Dynamical Networks. Physics Letters A, 372, 1601-1606. https://doi.org/10.1016/j.physleta.2007.10.020

[14] Yang, X.S. and Cao, J.D. (2014) Hybrid Adaptive and Impulsive Synchronization of 
uncertain Complex Networks with Delays and General Uncertain Perturbations. Applied Mathematics and Computation, 227, 480-493. https://doi.org/10.1016/j.amc.2013.11.025

[15] Li, N. and Cao, J.D. (2016) Passivity and Robust Synchronization of Switched Interval Coupled Neural Networks with Time Delay. International Journal of Systems Science, 47, 2827-2836. https://doi.org/10.1080/00207721.2015.1029570

[16] Li, S.Q., Peng, X.Y., Tang, Y. and Shi, Y.J. (2018) Finite-time Synchronization of Time-Delayed Neural Networks with Unknown Parameters via Adaptive Control. Neurocomputing, 308, 65-74. https://doi.org/10.1016/j.neucom.2018.04.053

[17] Zhang, J. and Gao, Y.B. (2017) Synchronization of Coupled Neural Networks with Time-Varying Delay. Neurocomputing, 219, 154-162.

https://doi.org/10.1016/j.neucom.2016.09.004

[18] Wu, W., Zhou, W.J. and Chen, T.P. (2009) Cluster Synchronization of Linearly Coupled Complex Networks under Pinning Control. IEEE Transactions on Circuits and Systems I: Regular Papers, 56, 829-839.

https://doi.org/10.1109/TCSI.2008.2003373

[19] Chen, L., Wu, L. and Zhu, S. (2008) Synchronization in Complex Networks by Time-Varying Couplings. European Physical Journal D, 48, 405-409. https://doi.org/10.1140/epjd/e2008-00113-4

[20] Huang, L.H., Wang, Z.Y., Wang, Y.N. and Zuo, Y. (2009) Synchronization Analysis of Delayed Complex Networks via Adaptive Time-Varying Coupling Strengths. Physics Letters A, 373, 3952-3958. https://doi.org/10.1016/j.physleta.2009.08.063

[21] Liu, X. and Chen, T. (2018) Finite-Time and Fixed-Time Cluster Synchronization with or without Pinning Control. IEEE Transactions on Cybernetics, 48, 240-252. https://doi.org/10.1109/TCYB.2016.2630703

[22] Liu, X.Y., Ho, D.W.C., Song, Q., et al. (2019) Finite/Fixed-Time Pinning Synchronization of Complex Networks with Stochastic Disturbances. IEEE Transactions on Cybernetics, 49, 2398-2403. https://doi.org/10.1109/TCYB.2018.2821119

[23] Zhang, W.L., Yang, X.S., Xu, C., et al. (2018) Finite-time Synchronization of Discontinuous Neural Networks with Delays and Mismatched Parameters. IEEE Transactions on Neural Networks and Learning Systems, 29, 3761-3771. https://doi.org/10.1109/TNNLS.2017.2740431

[24] Feng, J.W., Li, N., Zhao, Y., Xu, C. and Wang, J.Y. (2017) Finite-time Synchronization Analysis for General Complex Dynamical Networks with Hybrid Couplings and Time-Varying Delays. Nonlinear Dynamics, 88, 2723-2733.

https://doi.org/10.1007/s11071-017-3405-5

[25] Wu, X.J. and Lu, H.T. (2012) Projective Lag Synchronization of the General Complex Dynamical Networks with Distinct Nodes. Communications in Nonlinear Science and Numerical Simulation, 17, 4417-4429. https://doi.org/10.1016/j.cnsns.2012.03.019

[26] Xiang, J. and Chen, G.R. (2007) On the V-Stability of Complex Dynamical Networks. Automatica, 43, 1049-1057. https://doi.org/10.1016/j.automatica.2006.11.014

[27] Wang, Y.L. and Cao, J.D. (2013) Cluster Synchronization in Nonlinearly Coupled Delayed Networks of Non-identical Dynamic Systems. Nonlinear Analysis. Real World Applications, 14, 842-851. https://doi.org/10.1016/j.nonrwa.2012.08.005

[28] Li, Q.B. (2018) Synchronization Analysis and Control of Several Complex Dynamical Networks. Ph.D. Thesis, University of Science and Technology Beijing, Beijing.

[29] Cai, S.M., He, Q.B., Hao, J.J. and Liu, Z.R. (2010) Exponential Synchronization of 
Complex Networks with Nonidentical Time-Delayed Dynamical Nodes. Physics Letters A, 374, 2539-2550. https://doi.org/10.1016/j.physleta.2010.04.023

[30] Liu, H., Lu, J.A. and Lu, J.H. (2009) Structure Identification of Uncertain General Complex Dynamical Networks with Time Delay. Automatica, 45, 1799-1807. https://doi.org/10.1016/j.automatica.2009.03.022

[31] Wang, L., Chen, S.Y. and Wang, Q.G. (2015) Eigenvalue Based Approach to Bounded Synchronization of Asymmetrically Coupled Networks. Communications in Nonlinear Science and Numerical Simulation, 22, 769-779. https://doi.org/10.1016/j.cnsns.2014.08.022

[32] Qian, C.J. and Li, J. (2005) Global Finite-Time Stabilization by Output Feedback for Planar Systems without Observable Linearization. IEEE Transactions on Automatic Control, 50, 885-890. https://doi.org/10.1109/TAC.2005.849253

[33] Bhat, S.P. and Bernstein, D.S. (2000) Finite-time Stability of Continuous Autonomous Systems. SIAM Journal on Control and Optimization, 38, 751-766. https://doi.org/10.1137/S0363012997321358 